\title{
NEAR-RESONANT NONLINEAR OPTICAL PROPERTIES OF PSEUDOISOCYANINE J-AGGREGATES IN LANGMUIR-BLODGETT LAYERS
}

\author{
K.-H. FELLER ${ }^{\mathrm{a}, *}$, R. GADONAS ${ }^{\mathrm{b}}$, A. PUGZLYS ${ }^{\mathrm{b}}$ \\ and D. MÖBIUS ${ }^{\mathrm{c}}$
}

${ }^{a}$ Fachhochschule Jena, Fachbereich Medizintechnik/Physikalische Technik, Tatzendpromenade 1b, D-07745 Jena, Germany; ${ }^{\mathrm{b}}$ Vilnius University, Laser Research Center, Sauletekio ave. 10. LT-2054 Vilnius, Lithuania; ${ }^{\mathrm{c}}$ Max-PlanckInstitut für biophysikalische Chemie, Am Faßberg, D-37077 Göttingen, Germany

(Received 17 December 1996)

The nonlinear optical properties of pseudoisocyanine J-aggregates in Langmuir-Blodgett films have been investigated by means of degenerate four wave mixing (DFWM) process. The conversion efficiency of the DFWM signal and the estimated third order susceptibility were found to be comparable with those of pseudoisocyanine J-aggregates in solution under comparable resonance conditions. The intensity dependence of the converted (DFWM) signal is interpreted as resulting from different processes including saturation, exciton-exciton annihilation and secondary processes of dynamical disorder.

Keywords: Langmuir-Blodgett layers; excitonic processes; J-aggregates; DFWM; third order susceptibility

\section{INTRODUCTION}

The main advantage of organic nonlinear materials is that they have large susceptibilities enabling high efficiency of a nonlinear optical process at relatively low intensity together with fast response to the incoming signal [1]. Such materials can be used for ultrafast switching of light signals. Furthermore, if the material response under consideration additionally depends in a defined manner upon the

\footnotetext{
*Author to whom correspondence should be addressed.
} 
signal level (e.g., the light intensity applied) it is possible to use the temporal discrimination enabling new kinds of data processing in the ultrafast regime [2]. Such nonlinear materials are of high need for discriminative optical computing which is similar to information processing and information transport in life processes. However, the nonlinear optical phenomena in organic materials which possess strongly intensity depended responses are not well understood until now and are far away from technical use. The investigation of cooperative processes like those proceeding in $\mathbf{J}$-aggregates of polymethine dyes under defined and properly alignable conditions very efficiently contributes to the better understanding and future use of such materials for technical purposes including solid layers of organic molecules.

From the point of view of molecular engineering thin solid molecular films seem to be the most promising candidates for future nonlinear optical devices. For this purpose the fabrication technique of thin films of organic materials with high nonlinearity is often emphasized. The Langmuir-Blodgett (LB) technique [3] is a powerful method for producing thin molecular films consisting of multiple monomolecular layers with well ordered structure.

Compared to other thin layer techniques the Langmuir-Blodgett technique offers the unique possibility to align the structure on a molecular level. Special devices can be relatively easy designed by introducing molecules sensitive to a certain property into the layer structure, changing the molecular substituents which control the intermolecular distances, the angular distribution, the symmetry and other parameters important for optical nonlinearity [3, 4]. Furthermore, recent progress in (LB) technology enables formation of twodimensional J-aggregate mono- and multilayers on glass substrates [1, 4]. Such films possess spectral peculiarities close to those of selfaggregated polymethine dyes in aqueous solution and the nonlinear optical properties characteristic for molecular organic materials with highly delocalized $\pi$-electron system. Study of primary processes of photoexcited LB films of dye aggregates as well as investigation of nonlinear properties of LB mono- and multilayers is of great importance aiming to the understanding of the relationship between the optical nonlinearity, relaxation rates and mechanisms, structure and dimensionality of molecular assembly [5-9]. 
On the other hand, the dynamics of Frenkel excitons under restricted geometries is one of important fields of fundamental research increasingly exploited with the understanding of the role of confinement in the giant nonlinearities and their time responses [10-12]. J-aggregates of polymethine dyes are the self organizing systems well suitable for the study of the dynamics of 1-D confined excitons [12-14]. J-aggregates are linear chains of coupled chromophores with high size-enhanced resonance third order non-linear optical susceptibility [15]. Such features of aggregate excited state decay as Auger processes may be useful for non-linear optical applications as the mechanism preventing the absorption saturation and enabling high energy exchange and fast responses in various non-linear optical experiments is of great importance.

The main factor which limits seriously the investigation of the nonlinear properties of LB films as well as practical application of them is limited photostability [16]. Due to this reason until now there are only scarce experimental investigations of nonlinearity in LB films published $[6,7,9]$.

The photo-decomposition of polymethine dyes in LB films is the light intensity dependent process caused by space restriction of intramolecular torsional freedom, limited rate of exchange of vibrational energy with the surrounding and the absence of a solvent shell where the excess energy can be transferred to [6]. Although the preliminary results obtained do not give detailed understanding of the photo-decomposition mechanisms they can be used for minimization of the influence of the layer destruction to the results of the nonlinear optical experiments. Experimental study of the photo-decomposition of the LB films have shown that within some limits of the irradiation dose the photodestruction does not influence substantially the spectral characteristics of the layer [16]. Slight decrease in optical density of the sample reflects quantitative photochemical changes without appearance of qualitatively new features in spectral properties of the molecular layers. It can be therefore considered that within the same limits the nonlinear response of the system does not change much. This makes the preliminary investigation of the nonlinear optical processes in LB films possible. Nevertheless, systematic error in the case of nonlinear optical measurements in LB films increases.

We performed an experimental study of J-aggregates of 1-methyl-1'octadecyl-2,2'-cyanine ( pseudoisocyanine, PIC-C ${ }_{18}$ ) perchlorate in LB 
films in order to characterize the films as nonlinear optical media for the first time. Experiments have been done to estimate the third order susceptibility, the time scale behavior of the resulting light signal, its intensity and wavelength dependence. Due to low photostability there are only scarce experimental data on the decay of excited states in LB films containing $\mathbf{J}$-aggregates at high excitation intensities [17]. The present knowledge of the photophysics and nonlinear optical properties of $\mathrm{J}$-aggregates is limited to solutions $[10-15,18-21]$ and adsorbed aggregates on dispersed particles [22, 23]. Furthermore, the decomposition of the film occurs under illumination which influences the measured quantities in a complicated way which is not well understood. Due to this reason the interpretation of the data obtained is rather qualitative and is done assuming that the ultrafast photoprocesses in J-aggregates in LB films are similar to those in solution.

\section{EXPERIMENTAL}

Nonlinear optical properties of LB film of 1-methyl, l'-dioctadecyl-2, 2'-cyanine perchlorate (PIC-C 18 ) of 40 monomolecular layers (for absorption spectrum of the PIC layer system see Fig. 2) were investigated by using degenerate four wave mixing (DFWM) experiments in forward or so-called BOXCARS geometry (see Fig. 1) of the interacting beams. Output of the hybrid $\mathrm{LiNbO}_{3}-\mathrm{KDP}$ OPO (for a detailed description of the system see [24]) was split into three beams and focused on the sample into a spot of $3 \mathrm{~mm}^{2}$. The pulse duration was approximately $7 \mathrm{ps}$ and the energy in all three beams was approximately $40 \mu \mathrm{J}$, ensuring input intensity of $10^{14} \mathrm{phot} / \mathrm{cm}^{2}$ per pulse. Measurements were performed in the spectral region $575-595 \mathrm{~nm}$.

During the measurement of mixing efficiency for estimation of nonlinear susceptibility as well as the time responses of the $\chi_{1111}^{(3)}$ component the every data point was obtained as an average over 100 shots. At the intensities used the photobleaching of the sample during the measurement was less than $10 \%$. This additionally results in slight anisotropy at the long wavelength tail of the J-band but not in considerable change of the shape of aggregate absorption. In case of measurements of the intensity dependence of the DFWM signal where 


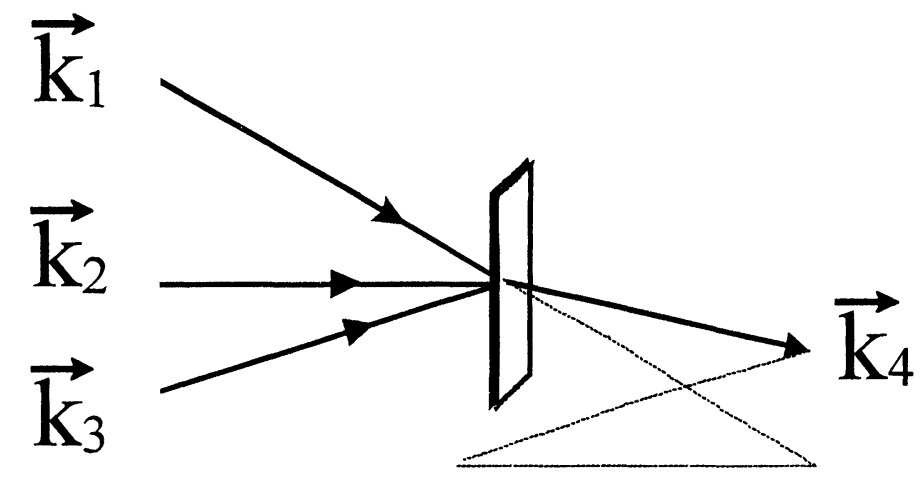

FIGURE 1 Beam arrangement in DFWM experiments in BOXCARS geometry. $\vec{k}_{1}, \vec{k}_{2}, \vec{k}_{3}$ are the interacting beams, and $\vec{k}_{4}=\vec{k}_{1}-\vec{k}_{2}+\vec{k}_{3}$ is the diffracted phase conjugated beam.

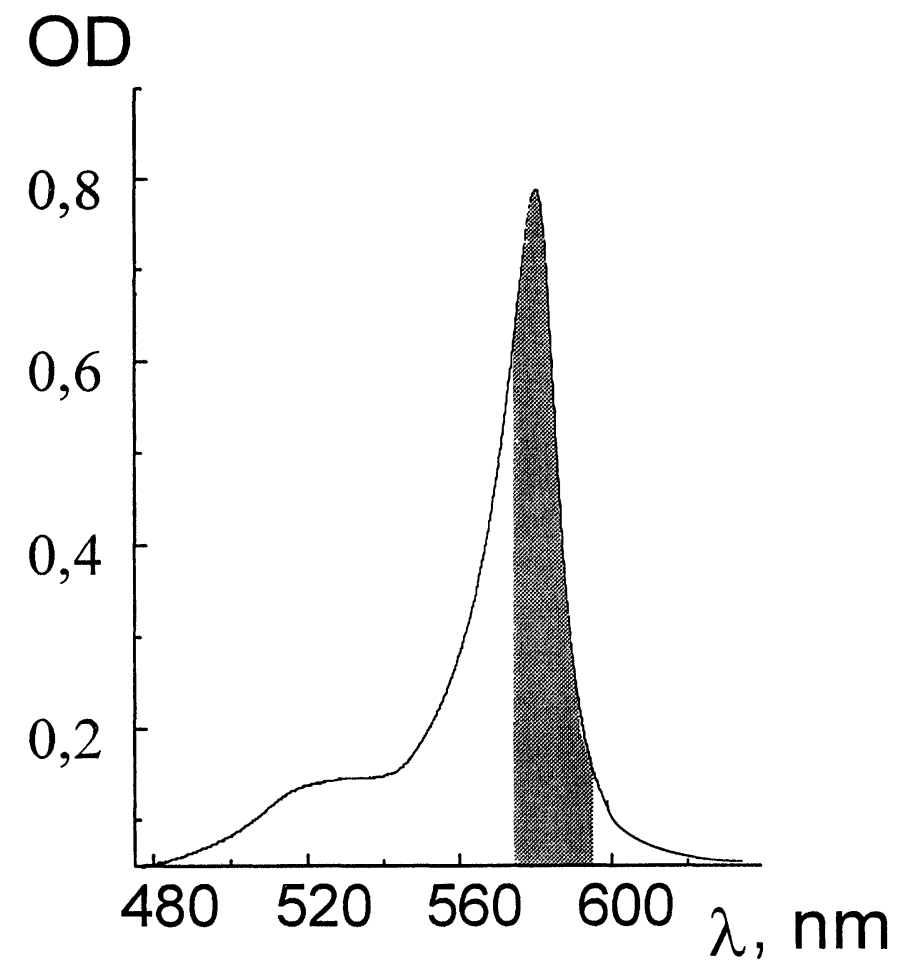

FIGURE 2 Absorption spectrum of PIC-C $\mathrm{C}_{18} \mathrm{~J}$-aggregates in a Langmuir-Blodgett film of 40 layers. Investigated spectral region is marked. 
much more (than 100) pulses were applied the sample was continuously moved in the plane perpendicular to the direction of incident beams. It ensured the replacement of the irradiated area of the sample after every single shot. The moving of the sample ensured the similar (corresponding to 100 pulses) integral irradiation dose per unit area. The decrease of the optical density of the irradiated part of the sample (about $0.5 \mathrm{~cm}^{2}$ ) during the whole mesurement was about $12 \%$.

\section{RESULTS AND DISCUSSION}

The intensity dependence of DFWM signal in J-aggregated LB film has been measured at exact timing of all three interacting pulses in the sample. The results are depicted in Figure 3. The dependence has been measured at $585 \mathrm{~nm}$ (detuning of $\approx 7 \mathrm{~nm}$ from the exact resonance or from the maximum of the $\mathbf{J}$-band). Intensity of pulses interacting in the LB film was changed in the range corresponding to one absorbed photon per $110 \div 7$ monomer molecules per 7 ps pulse at wavelength $585 \mathrm{~nm}$. Therefore, it allows to conclude that at intensities used in our experiments the annihilation affects substantially the nonliner optical process in solution and is very probable in LB films.

Figure 3 shows that in an LB film the intensity dependence of the DFWM signal does not follow strictly the third order intensity dependence which is characteristic for off resonance case of DFWM and has been shown for PIC J-aggregates in solution [15, 18]. The third order dependence of the DFWM signal intensity on the input is observed only at applied intensities corresponding to less than one absorbed photon per 30 monomer molecules.

Surprizingly, the logarithmic plot results not in a straight line dependence with one power over the whole intensity range as for all investigated wavelengths in $\mathrm{J}$-aggregate solutions received [13, 14]. At low intensities the ascent of the curve is 3.0 as expected for low intensities (annihilation and saturation free conditions) whereas at higher intensities the ascent decreases to 1.6. It seems that a much slower annihilation (and higher static disorder) under geometry restricted conditions in LB films is responsible for that behavior [25]. Additionally, it can be supposed that the complex character of the time dependence of DFWM signal is caused by the destructive super- 


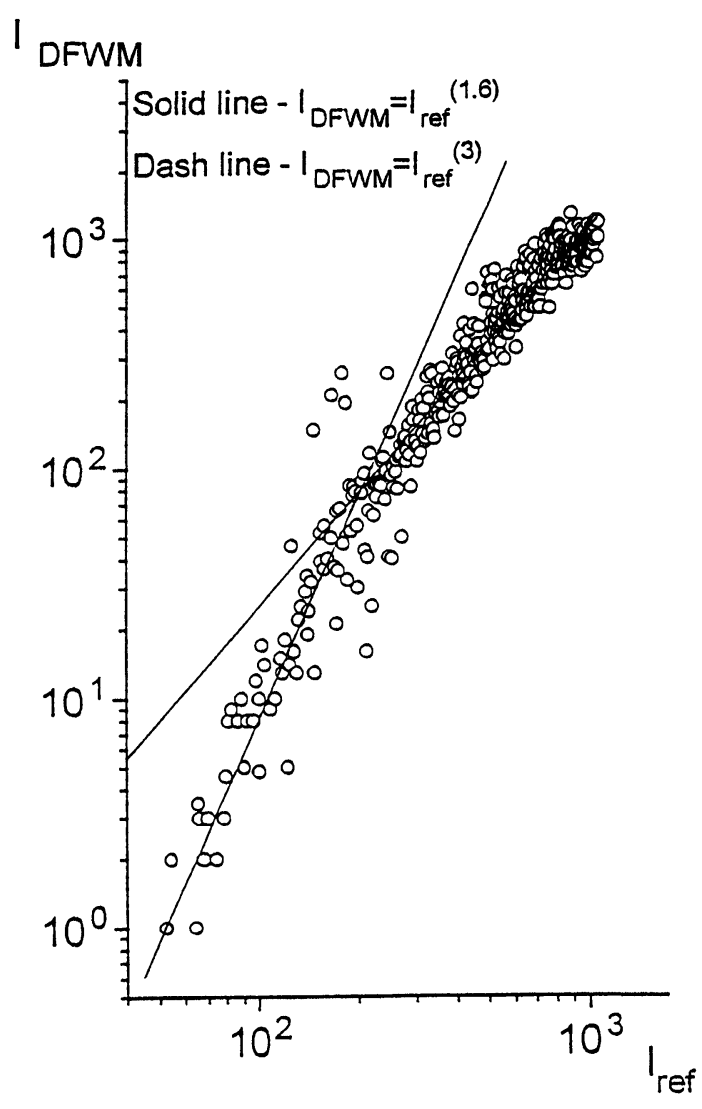

FIGURE 3a Intensity dependence of DFWM signal of PIC-C ${ }_{18} \mathrm{~J}$-aggregates in a Langmuir-Blodgett film of 40 layers at wavelength $585 \mathrm{~nm}$.

position of the gratings originated from exciton population and disordering [13]. In J-aggregates in solution at excitation intensity corresponding to 1 absorbed photon per 7 molecules, reversible disordering of the $\mathrm{J}$-aggregate occurs which is manifested by the delayed transient absorption arising in the long wavelength tail of the $\mathrm{J}$-band [18]. For comparison of the relaxation kinetics in cases of $\mathbf{J}$ aggregates in solution and in LB films it should be mentioned that in the case of LB films the transfer of vibrational excitation to the surrounding is slower than in solution. In this case delayed transient absorption characterisitic for $\mathrm{J}$-aggregates in solution at high 


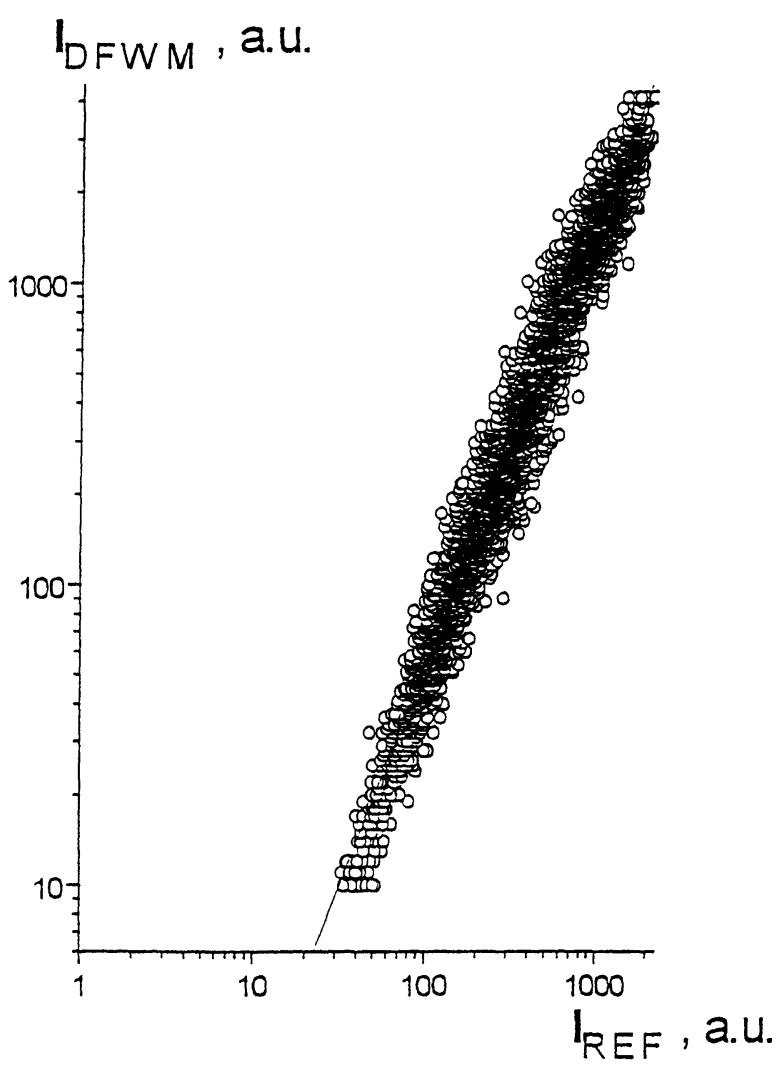

FIGURE 3b Intensity dependence of DFWM signal of PIC J-aggregates in aqueous solution at a concentration of $10^{-2} \mathrm{M}$ at wavelength $575 \mathrm{~nm}$ (in exact resonance) (taken from ref. [15]).

excitation intensities can have different character. Due to this reason direct comparison of the relaxation kinetics of $\mathrm{J}$-aggregates in solution and in LB films remains a difficult task.

As discussed in $[15,18,26,27]$ exciton-exciton annihilation plays an important role in case of a $\mathrm{J}$-aggregate solution resulting in deviation from the third order power dependence of DFWM signal on intensity near resonance. The fast annihilation process is expected to occur at high intensity in LB films also. This assumption is supported by observation of the saturation of the integrated fluorescence intensity under excitation with picosecond pulses of relatively low intensity [16]. 
The annihilation is mediated by energy migration, which could be very fast in an LB film since the two-dimensional packing decreases the average distance between delocalized excitations in an aggregate domain and the different aggregate domains become closer each other which can enable interdomain transfer. On the other hand, the existence of a resonant dipole transition to a higher excited state is necessary for annihilation. Although in solution there exists a blueshifted transient absorption of the excited state, the spectra of excited J-aggregates in LB films have never been measured. However, the theoretical analysis of the aggregate difference spectrum [19] presumes that blue-shifted transient absorption for every liner aggregate possessing the J-type transition. Taking this into account, annihilation can be expected to be responsible for the deviation from third order intensity dependence in J-aggregated LB films and for the decrease of DFWM efficiency at high excitation intensities. On the other hand, as discussed in $[26,27]$, the exciton-exciton annihilation in J-aggregates reduces significantly full saturation of exciton population and is the process enabling resonance DFWM at high intensity of interacting light pulses.

The relaxation kinetic of the DFWM signal (polarizations of all interacting beams were parallel) was found to have complex character. As a first step the decay rates were evaluated using two-exponential fitting resulting in two characteristic relaxation times of $15 \mathrm{ps}$ and $400 \mathrm{ps}$ (Fig. 4). This is not fully correct because in case of annihilation at least the short component becomes non-exponential (Paillotin's approach [28] is a better description of the temporal behavior under such conditions), but on the level of precision of data received, more sophisticated models as [28] gave no better fits to the experimental data.

Taking into account the data known from J-aggregates in solution $[29,18]$ and the aspect that the incorporation of J-aggregates in solid films increases the annihilation-free lifetime the two components are supposed due to the annihilation (short component) and due to the annihilation-free lifetime of the J-aggregate (long lifetime).

Let us for a first step estimate the DFWM efficiency in J-aggregates of PIC in LB films. The values obtained for the efficiency

$$
\operatorname{eff}_{\mathrm{DFWM}}=\frac{I_{4}}{I_{1}+I_{2}+I_{3}}
$$




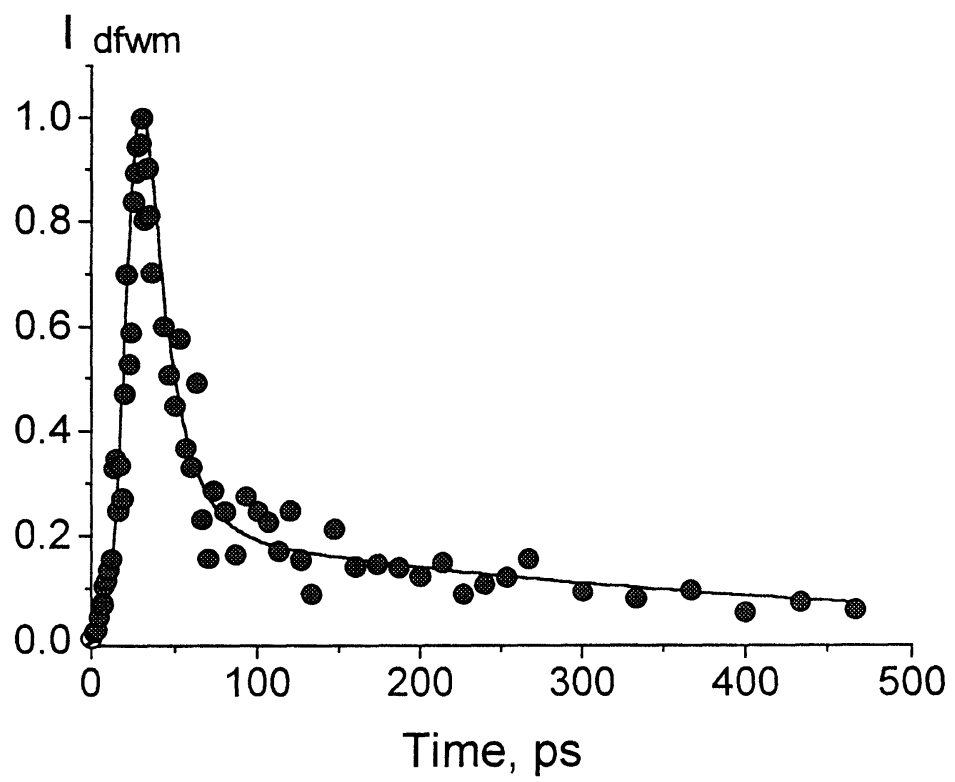

FIGURE 4 Relaxation of DFWM signal of PIC-C C $_{18}$ in a Langmuir-Blodgett film (40 layers) at the wavelength $585 \mathrm{~nm}$.

are comparable to those obtained for $\mathrm{J}$-aggregates in solution in 0.2 mm cuvette at concentration $10^{-2} \mathrm{M}$ [14]. Furthermore, the spectrum of the DFWM efficiency is well correlated with the absorption spectrum (see Fig. 5). It shows that the nonlinearity of PIC Jaggregates in LB film near resonance is mainly determined by real excitations like in J-aggregates in solution [14]. From the results the similar ultrafast processes contributing to the nonlinearity of $\mathrm{J}$ aggregates can be expected in both, solution and LB film.

From the measured DFWM efficiency the $\left|\chi^{(3)}\right|$ value of $(3 \pm 1.3) \times 10^{-7}$ esu was estimated for the LB film of PIC J-aggregates at $590 \mathrm{~nm}$ by using equation [30]:

$$
\left|\chi^{(3)}\right|=\frac{c n^{2}}{32 \pi^{2}}\left(\frac{\lambda w_{o} \tau}{2 l_{\mathrm{eff}}}\right) \sqrt{\frac{45}{8} \frac{E_{4}}{E_{1} E_{2} E_{3}}}
$$

which can be used for estimation of the value of third order nonliner susceptibility in the near-resonance case by taking into account the 


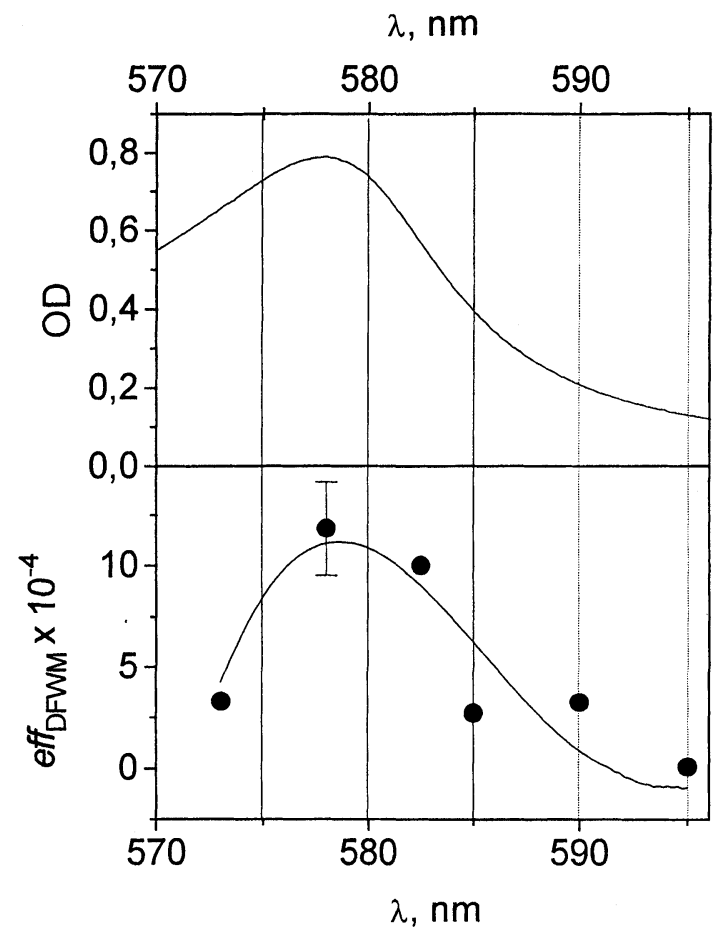

FIGURE 5 Spectra of DFWM efficiency (bottom) and absorption (top) of PIC-C $\mathrm{C}_{18} \mathrm{~J}$ aggregates in a Langmuir-Blodgett film.

properties of the interacting light pulses by the wavelength $\lambda$, the beam waist in the focus $w_{0}$, the pulse duration $\tau$, as well as the refractive index of the material $n$ and the effective thickness of the sample $l_{\text {eff }}$ which allows to eliminate the influence of the absorption:

$$
l_{\text {eff }}=\frac{1-\exp (-\alpha l)}{\alpha}
$$

There $\alpha$ is the absorption coefficient of the sample, 1 is the thickness of the sample. $E_{1}, E_{2}$, and $E_{3}$ in the equation (2) are the energies of the interacting beams. $E_{4}$ is the energy of the diffrated beam.

For comparison of nonlinear susceptibilities in J-aggregates in LB film and solution we have calculated $\left|\chi^{(3)}\right|$ value for bulk material i.e. for solid containing only PIC molecules. The LB film of J-aggregates 
contains not only the "nonlinearly active" PIC molecules but also long aliphatic chains $\mathrm{C}_{18} \mathrm{H}_{37}$ between the layers the contribution to the nonlinearity of which is negligible. The thickness of the interlayer can be estimated as the length of the aliphatic chain of $\sim 25 \AA$. Taking into account that the height of molecule of 1,1'-diethyl-2, 2-cyanine chloride is about $1.5 \AA$, it can be deduced that, active "molecules take only $\sim 6 \%$ of the volume of the LB film. This allows to estimate the $\left|\chi^{(3)}\right|$ value for highest packing density of "active" molecules (nonlinear susceptibility of bulk material) of $(5.1 \pm 2.2) \times 10^{-6}$ esu at $590 \mathrm{~nm}$.

The above estimated value of $\left|\chi^{(3)}\right|$ give the possibility to compare the results obtained for PIC J-aggregates in solution and in LB film (see Tab. I). It should be noted that the optical density of PIC Jaggregates in solutions at concentration $10^{-2} \mathrm{M}$ at $590 \mathrm{~nm}$ is 0.18 . This is close to that of a 40 layer film at the same wavelength $\sim 0.2$. On the other hand, the total number of monomer molecules in the irradiated volume which is limited to by area $3 \mathrm{~mm}^{2}$ and thickness of the sample (40 monolayers in the case of LB film and $0.2 \mathrm{~mm}$ in the case of solution) is estimated to be $3.6 \times 10^{15}$ and $2.5 \times 10^{14}$ for solution and LB film, respectively. The difference means different extinction coefficients for PIC in solution and LB film due to $5 \mathrm{~nm}$ red shift and approximately two times broader J-band in the case of LB film. Taking into account that the nonlinearity decreases very strongly going out of resonance, the higher value of $\left|\chi^{(3)}\right|$ at highest packing density in the case of LB film with respect to that in solution $(2 \pm 1) \times 10^{-6}$ esu, estimated at $590 \mathrm{~nm}$ from data obtained in DFWM

TABLE I Comparison of third order nonlinear susceptibilities $\left|\chi^{(3)}\right|$ of pseudoisocyanine J-aggregates in solution and in Langmuir-Blodgett layers measured at the used concentration or layer thickness and calculated for highest packing density.

The values for $\mathrm{J}$-aggregates in aqueous solution were taken from $[14,15]$

\begin{tabular}{lcccc}
\hline Sample & $c($ mol $/ l)$ & $\lambda(\mathrm{nm})$ & measured $\left|\chi^{(3)}\right|$ & $\begin{array}{c}\text { calculated }\left|\chi^{(3)}\right| \text { for } \\
\text { highest packing density }\end{array}$ \\
\hline $\begin{array}{l}\text { J-aggregates } \\
\text { in solution }\end{array}$ & $5 \cdot 10^{-4}$ & 579 & $(1.2 \pm 0.5) \cdot 10^{-11} \mathrm{esu}$ & $(5 \pm 4) \cdot 10^{-7} \mathrm{esu}$ \\
$\begin{array}{l}\text { J-aggregates } \\
\text { in solution }\end{array}$ & $10^{-2}$ & 590 & $(3 \pm 1) \cdot 10^{-11} \mathrm{esu}$ & $(7 \pm 4) \cdot 10^{-8} \mathrm{esu}$ \\
$\begin{array}{l}\mathrm{J} \text {-aggregates } \\
\text { in LB film }\end{array}$ & - & 590 & $(1.5 \pm 1) \cdot 10^{-7} \mathrm{esu}$ & $(2 \pm 1) \cdot 10^{-6} \mathrm{esu}$ \\
\hline
\end{tabular}


experiments with $\mathrm{J}$-aggregate solution of concentration $10^{-2} \mathrm{M}$ ) can be explained (see Tab. I). Furthermore, it should be mentioned that equation (2) can be correctly used only in case of third order intensity dependence (saturation effects do not take place in the DFWM process) as well as when absorption of the sample is not very high. The above estimated value of $\left|\chi^{(3)}\right|$ in J-aggregated LB film is reduced by influence of annihilation and limited photostability of the sample. At excitation intensity $\sim 0.2 \mathrm{GW} / \mathrm{cm}^{2}$ which corresponds to one absorbed photon per 7 monomer molecules in the case of the J-aggregated LB film annihilation and secondary vibrational processes contribute to the decrease of the efficiency of DFWM stronger than in the case of solution, where the same excitation intensity corresponds only to one absorbed photon per 120 monomer molecules (concentration of the sample is much lower). Also the photodecomposition of the $\mathrm{J}$ aggregates in LB films not only increases the systematic error of the measurements but also contributes to the decrease of DFWM efficiency and of the value of $\left|\chi^{(3)}\right|$. The photodecomposition of $\mathrm{J}$ aggregates starts from irreversible deaggregation of the longest ones. On the other hand, it is shown in [10] that nonlinear susceptibility of Jaggregates is very sensitive to the physical size of the aggregate and depends quadratically on the number of monomer units joined into the aggregate. From that can be supposed, that the nonlinear optical susceptibility of $\mathrm{J}$-aggregates has weak tendency to increase by placing the aggregates into a two-dimensional restricted area.

\section{CONCLUSION}

We investigated near-resonant nonlinear properties of $\mathrm{J}$-aggregated LB films by the DFWM technique. The estimated value of $\left|\chi^{(3)}\right|$ for Jaggregates in LB film is comparable with that calculated for highest packing density from $\left|\chi^{(3)}\right|$ value obtained for J-aggregates in solution. On the other hand, the value is reduced due to exciton-exciton annihilation and limited photostability of the sample. The temporal behavior as well as intensity dependence of DFWM process of $\mathrm{J}$ aggregates in LB layers is qualitatively similar to that in solution and can be explained by influence of annihilation and secondary vibrational energy dynamics to the nonlinear optical response. 


\section{Acknowledgements}

The work has been partly supported by the Deutsche Forschungsgemeinschaft within the Sonderforschungsbereich 196 "Physik und Chemie optischer Schichten". R. G. and A. P. acknowledge the International Science Foundation for partial support of equipment maintenance. We thank Prof. W. Grahn (TU Braunschweig) for a gift of PIC-C ${ }_{18}$. Many valuable discussions with Prof. A. Piskarskas (Vilnius University) are gratefully acknowledged. Mrs. E. Kielmann we thank for technical assistance during preparation of the LangmuirBlodgett films.

\section{References}

[1] Chemla, D. S. and Zyss, J. (1987). Nonlinear Optical Properties of Organic Molecules and Crystals, Academic Press, Orlando.

[2] Sasaki, F. and Kobayashi, Sh. (1993). Appl. Phys. Lett., 63, 2887.

[3] Fuchs, H., Ohst, H. and Prass, W. (1994). Adv. Mater., 3, 10.

[4] Feller, K.-H. and Gadonas, R. (1994). Lith. Journal Physics, 34, 163.

[5] Feller, K.-H., Gadonas, R., Krasauskas, V. and Pugzlys, A. (1993). Intern. J. Optoelectr., 8, 677.

[6] Kawaguchi, T. and Iwata, K. (1988). Thin Solid Films, 165, 323.

[7] Cnossen, T., Drabe, K.E. and Wiersma, D. A. (1992). J. Chem. Phys., 97, 4512.

[8] Terpstra, J., Fidder, H. and Wiersma, D. A. (1991). Chem. Phys. Lett., 179, 349.

[9] Marowsky, G., Chi, L. F., Möbius, D., Steinhoff, R., Shen, Y. R., Dorsch, D. and Rieger, B. (1988). Chem. Phys. Lett., 147, 420.

[10] Spano, F. and Mukamel, S. (1989). Phys. Rev., A 40, 5783.

[11] Spano, F. and Mukamel, S. (1991). J. Chem. Phys., 95, 7526.

[12] Knoester, J. (1995). Adv. Mater., 7, 500.

[13] Gadonas, R., Feller, K.-H., Jonusauskas, G., Oberle, G., Pugzlys, A. and Rulliere, C. (1997). J. Chem. Phys., 106 (20), (in press).

[14] Gadonas, R., Feller, K.-H. and Pugzlys, A. (1997). Laser Chemistry, 17, 1.

[15] Minoshima, K., Taiji, M., Misawa, K. and Kobayashi, T. (1994). Chem. Phys. Lett., 218, 67.

[16] Feller, K.-H., Pugzlys, A., Gadonas, R., Baltuska, A. and Möbius, D., Thin Solid Films (submitted).

[17] Dorn, H.-P. and Müller, A. (1988). Appl. Phys., B 43, 351.

[18] Sundström, V., Gillbro, T., Gadonas, R. and Piskarskas, A. (1988). J. Chem. Phys., 89, 2754.

[19] Gagel, R., Gadonas, R. and Laubereau, A. (1994). Chem. Phys. Lett., 3, 228.

[20] Ying Wang, J. (1991). Opt. Soc. Am., B 8, 981.

[21] Gadonas, R., Feller, K.-H. and Pugzlys, A. (1994). Opt. Comm., 112, 157.

[22] Kemnitz, K., Yoshihara, K. and Tani, T. (1990). J. Phys. Chem., 92, 3099.

[23] Quitevis, E. L., Horng, M.-L. and Chen, S.-Y. (1988). J. Phys. Chem., 92, 256.

[24] Pugzlys, A., Gadonas, R., Piskarskas, A. and Baltuska, A. (1995). Exp. Techn. Phys., 41, 57.

[25] Feller, K.-H, Gaizauskas, E., Gadonas, R. and Pugzlys, A., to be published.

[26] Gaizauskas, E., Feller, K.-H. and Gadonas, R. (1995). Opt. Comm., 118, 360.

[27] Gaizauskas, E. and Feller, K.-H., J. Phys. Chem., (submitted). 
[28] Paillotin, G., Swenberg, C. E., Breton, J. and Geacintov, N. E. (1979). Biophys. J., $25,513$.

[29] Brückner, V., Feller, K.-H. and Grummt, U.-W. (1990). Applications of timeresolved optical spectroscopy (Elsevier, North Holland, Amsterdam).

[30] Carter, G. M., Thakur, M. K., Chen, Y. J. and Hrynewich, J. V. (1985). Appl. Phys. Lett., 47, 457. 